07

\title{
Измерение тепловых характеристик фотоэлектрических концентраторных модулей
}

\author{
() А.В. Андреева, Н.Ю. Давидюк, Д.А. Малевский, П.В. Покровский, Н.А. Садчиков, А.В. Чекалин \\ Физико-технический институт им. А.Ф. Иофрфе РАН, \\ 194021 Санкт-Петербург, Россия \\ e-mail: chekalin@mail.ioffe.ru
}

Поступило в Редакцию 30 апреля 2020 г.

В окончательной редакции 2 июля 2020 г.

Принято к публикации 22 июля 2020 г.

Предложена методика для исследования тепловых свойств фотоэлектрических концентраторных модулей в помещении в затемненных условиях, сочетающая в себе процедуру нагрева модуля за счет пропускания постоянного тока и последующего быстрого измерения вольт-амперных характеристик, в течение которого температура фотоэлементов не успевает значительно измениться за счет теплоемкости материала. Были проведены сравнения характеристик шести типов концентраторных модулей, отличающихся конструкцией, размерами и материалами теплоотводов. Для концентраторных модулей больших размеров исследовано влияние пространственного расположения модулей на эффективность теплосброса.

Ключевые слова: концентраторные фотоэлектрические модули, тепловое сопротивление, $p-n$-переход, солнечные фотоэлементы, теплоотвод.

DOI: 10.21883/JTF.2021.02.50365.155-20

\section{Введение}

Эффективность преобразования фотоэлектрических концентраторных модулей сильно зависит от температуры на $p-n$-переходах $\left(T_{p-n}\right)$ входящих в состав модулей. Температура на $p-n$-переходах фотоэлементов зависит от преобразуемой оптической мощности, внутренних параметров фотоэлементов, условий отвода тепла и может сильно отличаться от температуры держателей, которая может быть измерена с помощью датчиков температуры. Наиболее распространенным методом непосредственного определения температуры $p$-n-переходов является оценка $T_{p-n}$ по значению напряжения холостого хода фотоэлемента $\left(V_{o c}\right)$. При этом даже при работе фотоэлементов в фактическом режиме холостого хода требуется знание соответствующего значения $V_{o c}$ при температуре окружающей среды без разогрева $p-n$-перехода и знание температурного коэффициента $\alpha$ для определения изменений температуры $p$ - $n$-перехода при изменении значений $V_{o c}$. Измерение значения $V_{o c}$ при температуре окружающей среды может осуществляться, например, с помощью механического затвора, быстро открывающего входную апертуру концентраторного модуля [1,2]. Но на практике при измерении этого параметра таким способом в модулях большой площади в натурных условиях возникают большие сложности из-за неконтролируемого нагрева элементов при подключении освещения. В работах [2-5] был предложен метод измерения температуры $p-n$-переходов фотоэлементов, работающих в режиме максимальной мощности. Для этого в исследуемом модуле с помощью электронного коммутатора происходило очень быстрое отключение внешней электрической нагрузки и производилось измерение броска напряжения при переходе из режима максимальной мощности в режим холостого хода. В этом случае измерения $V_{\text {ос }}$ при комнатной температуре путем механического открывания входной апертуры может быть заменено проведением несложных вычислений $[4,5]$. Температурный коэффициент $\alpha$ должен быть измерен в помещении на уровне отдельных элементов или тестируемого модуля в целом, с помощью имитаторов солнечного излучения $[2-4,6]$.

Предложенные методики позволяют оценить температуры $p-n$-переходов фотоэлемента в любом режиме эксплуатации концентраторного модуля в натурных условиях. Однако эти условия не являются стабильными, и температура сильно зависит от набора условий окружающей среды: солнечного спектра, интенсивности освещения, скорости ветра. В работе [6] для полноразмерных фотоэлектрических концентраторных модулей было предложено ввести универсальный параметр - эффективное тепловое сопротивление фотоэлемент-окружающая среда $-r_{t h}$. В натурных условиях его оценка требует измерений многих параметров в течение длительного времени. Расчет соответствующих параметров для модулей различной конструкции требует фильтрации результатов измерения по интенсивности, условиям освещения, спектральному составу излучения и скорости ветра с последующей компьютерной статистической обработкой полученных данных.

В настоящей работе предлагается методика, при которой теплофизические свойства фотоэлектрических концентраторных модулей определяются в помещении в затемненных условиях. Конкретные тепловые сопротивления вычисляются по тепловой нагрузке модулей при пропускании прямого тока. В качестве индикаторов 
температуры фотоэлементов служат значения прямого падения напряжения [7-9].

Использование значений прямого падения напряжения для оценки температуры $p$ - $n$-перехода является стандартным методом в исследованиях большинства электронных приборов [10], где используются температурные коэффициенты прямого падения напряжения $(\beta)$, полученные из экспериментов в термостатной камере. Тем не менее использование прямого тока для имитации тепловой нагрузки модуля вызывает дополнительные погрешности при расчете параметров за счет неравномерности распределения температуры. Причиной этого являются небольшие различия в падении напряжения на элементах и соединительных проводах при параллельнопоследовательном соединении в модулях групп элементов, установленных на многочисленных теплоотводах. При больших значениях тока его распределение по элементам становится неравномерным, что приводит к их неравномерному нагреву. Такая ситуация отсутствует при „нормальной“ работе модуля (при его засветке), когда осуществляется принудительное выравнивание поступающей солнечной энергии и генерируемого элементами фототока. Эту проблему при оценке $r_{t h}$ помогает преодолеть использование достаточно низких уровней прямого тока (самый низкий уровень ограничен приемлемой точностью измерений).

Другая проблема связана с перераспределением нагретого воздуха внутри корпуса модуля. В основном этого можно избежать в экспериментах внутри помещений, если исследовать модули меньшего размера. Для оценки влияния размеров модуля на величину параметра $r_{t h}$ в настоящей работе были исследованы характеристики полноразмерных и меньших по размеру модулей.

Предложенная нами методика сочетает в себе использование значений прямого падения напряжения для оценки температуры $p-n$-перехода при пропускании прямого тока с недавно разработанным подходом быстрого измерения вольт-амперных характеристик (BAX) при тепловой нагрузке, во время которого температура элемента сохраняется на постоянном уровне за счет теплоемкости материала [2-5].

Получаемые таким образом значения $r_{t h}$ могут быть определены с хорошей точностью, они незначительно зависят от величины прямого тока и, следовательно, могут быть использованы в качестве параметров, характеризующих теплофизические свойства фотоэлектрических концентраторных модулей различной конструкции.

Знание параметра $r_{t h}$ для конкретного модуля, измеренного в условиях лаборатории, позволяет сделать оценку максимального перегрева $\Delta T_{p-n}$ фотоэлементов в модуле при освещении его солнцем в отсутствии ветра в натурных условиях:

$$
\Delta T_{p-n}=k E r_{t h}\left(1-\eta_{P V}\right)\left[{ }^{\circ} \mathrm{C}\right]
$$

где $E-$ интенсивность солнечного излучения $\left[\mathrm{W} / \mathrm{m}^{2}\right]$, $r_{t h}-$ эффективное тепловое сопротивление фотоэлемент-окружающая среда $\left[{ }^{\circ} \mathrm{C} \cdot \mathrm{m}^{2} / \mathrm{W}\right], \eta_{P V}-$ эффективность фотоэлектрического преобразования исследуемого модуля, $k$ - оптическая эффективность линз Френеля. В формуле (1) множитель $\left(1-\eta_{P V}\right)$ характеризует ту часть падающей на фотоэлемент энергии, которая идет непосредственно на его разогрев, остальная же часть отводиться в полезную нагрузку.

\section{1. Методика определения тепловых сопротивлений фотоэлемент-окружающая среда}

Методика определения $r_{t h}$ в лабораторных условиях состоит из двух этапов. На первом этапе с использованием термостатической камеры записываются значения напряжения на сборках фотоэлементов при пропускании прямого тока в условиях фиксированных значений температур. На втором этапе сравниваются значения напряжения при одинаковых значениях тока модуля, нагретого за счет пропускании прямого постоянного тока, с соответствующими значениями напряжения при комнатной температуре и оценивается температура перегрева. Затем с учетом значений подводимой тепловой мощности и увеличения температуры вычисляется значение $r_{t h}$. Следует отметить, что при этом на корпусе модуля не используются датчики температуры.

В данной методике прямой ток выполняет две функции. С одной стороны, прямой ток при быстром изменении в момент включения/выключения индуцирует зависящее от времени изменение напряжения на клеммах модуля, которое используется в качестве индикатора усредненной температуры элементов при внешнем нагреве (этап 1). С другой стороны, прямой ток используется для термической нагрузки испытываемого модуля, при которой изменяется температура элементов в зависимости от величины постоянного тока и параметров системы рассеивания тепла (этап 2).

На этапе 1 при разных фиксированных значениях температур производится запись серии BAX в момент включения/выключения тока. Следует отметить, что пространственное положение модуля внутри термостата не имеет значения, поскольку измерения производятся после достижения полного теплового баланса всех частей модуля с окружающей средой камеры. При быстрых измерениях ВАX временные интервалы процессов нарастания/падения для постоянного тока были выбраны таким образом, чтобы изменения значений напряжения при одинаковых значениях тока за счет нагрева были бы незначительными. Этот интервал был выбран равным 50-60 $\mu \mathrm{s}$. В этих условиях быстрые измерения ВАХ не оказывали влияния на температуру элементов как при внешнем нагреве, так и при нагреве за счет постоянного тока. Затем был произведен расчет температурных коэффициентов напряжения модулей $\beta_{\text {mod }}$ при различных плотностях тока элементов. 
На этапе 2 модули были размещены в открытом пространстве лаборатории при контролируемой комнатной температуре. Для экспериментов было выбрано горизонтальное положение модулей с вертикальным направлением панели фотоприемников. Причина этого в том, что такая конфигурация является наиболее выгодной для выравнивания параметров тепловыделения для всех элементов в модуле. При исследовании тепловых характеристик полноразмерных фотоэлектрических концентраторных модулей были определены значения эффективных тепловых сопротивлений $r_{t h}$ при разном пространственном расположении модулей.

Значение вводимой электрической мощности $P_{E l}$ варьировалось, чтобы выявить влияние этого параметра при оценке $r_{t h}$. Для каждого значения мощности $P_{E l}$ была рассчитана величина температуры перегрева $p-n$ переходов элементов $\Delta T_{p-n}$ с использованием параметра $\beta_{\text {mod. }}$ При расчете $\Delta T_{p-n}$ используется формула

$$
\Delta T_{p-n}=\frac{V_{h o t}-V_{a m b}}{\beta_{\text {mod }}}\left[{ }^{\circ} \mathrm{C}\right],
$$

где $\beta_{\text {mod }}-$ температурный коэффициент напряжения модулей при заданной плотности тока $\left[-\mathrm{V} /{ }^{\circ} \mathrm{C}\right], V_{h o t}$ и $V_{a m b}$ - значения напряжения на модулях при одинаковых значениях тока, измеренные при быстрой записи $\mathrm{BAX}[\mathrm{V}]: V_{h o t}$ измеряется в состоянии теплового баланса при подведении электрической мощности $P_{E l}, V_{a m b}$ измеряется в первый момент испытания модулей, когда все детали находятся еще при температуре окружающей среды.

Для вычисления эффективного теплового сопротивления $r_{t h}$ была использована следующая формула:

$$
r_{t h}=\frac{S_{l e n s} \Delta T_{p-n}}{P_{E l}}\left[{ }^{\circ} \mathrm{C} \cdot \mathrm{m}^{2} / \mathrm{W}\right],
$$

где $S_{l e n s}-$ площадь линзовой панели в исследуемом модуле выбранной конструкции $\left[\mathrm{m}^{2}\right], \Delta T_{p-n}-$ температура перегрева $p-n$-переходов элементов $\left[{ }^{\circ} \mathrm{C}\right], P_{E l}-$ подводимая электрическая мощность [W].

\section{2. Конструкции исследуемых модулей}

Разработанный метод был применен в исследованиях тепловых характеристик концентраторных модулей разной конструкции. Для концентрации света были использованы линзы Френеля, изготовленные методом силикон-на-стекле и размещенные на линзовой панели. В фокусе каждой линзы были установлены трехпереходные $\mathrm{GaInP} / \mathrm{GaInAs} / \mathrm{Ge}$ фотоэлементы, напаянные на теплоотводы, закрепленные на приемной панели. Схематически сечение фрагмента модуля показано на рис. 1.

Для экспериментов по определению $r_{t h}$ в лабораторных условиях были изготовлены модули с размерами, соответствующими внутренним размерам имеющегося лабораторного термостата.

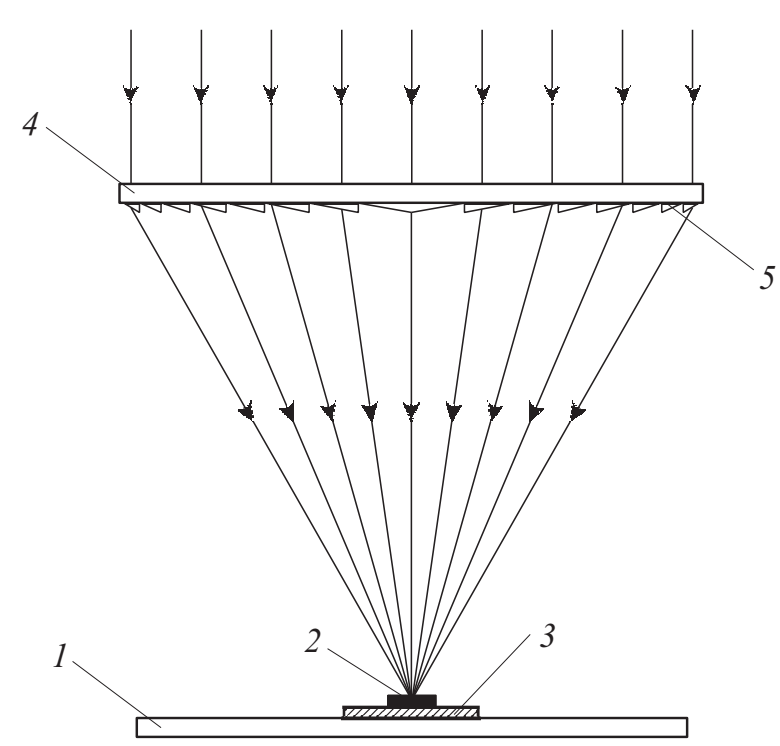

Рис. 1. Схематическое изображение сечения фрагмента фотоэлектрического концентраторного модуля: 1 - стеклянное основание, 2 - фотопреобразователь, 3 - теплоотводящее основание, 4 - стеклянная основа панели линз Френеля, 5 - профиль линз Френеля.

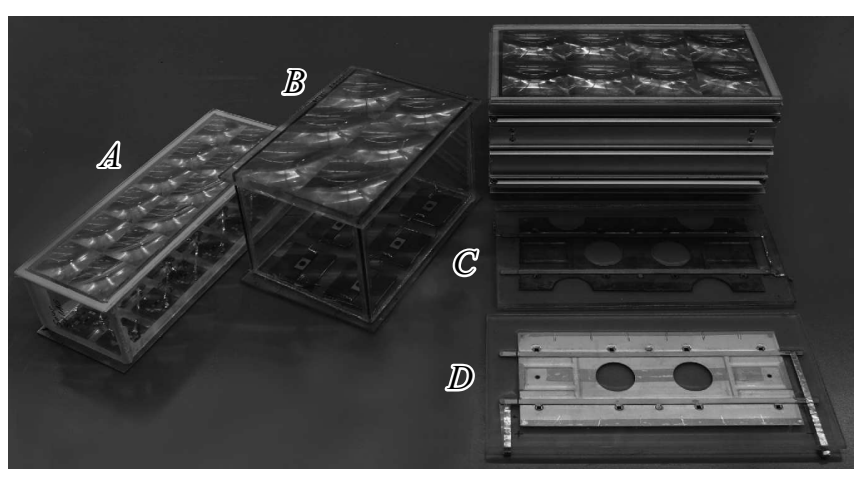

Рис. 2. Четыре типа фотоэлектрических концентраторных модулей $(A, B, C$ и $D)$ с различными конструкциями теплоотводов, используемыми в экспериментах по определению $r_{t h}$ в лабораторных условиях.

Были исследованы также тепловые характеристики полноразмерных модулей, разработанных для размещения на системах слежения за Солнцем в натурных условиях.

Среди небольших модулей в качестве объектов исследований были использованы четыре типа концентраторных модулей, отличающихся деталями систем рассеивания тепла. Изображение исследуемых модулей представлено на рис. 2.

Модуль типа $A$ содержал линзовую панель, состоящую из двенадцати линз Френеля размерами $40 \times 40 \mathrm{~mm}$ с фокусным расстоянием $70 \mathrm{~mm}$. Фотоэлементы размерами $3 \times 3.5 \mathrm{~mm}$ были напаяны на изолированные площадки алюмооксидных печатных плат, которые, в свою 


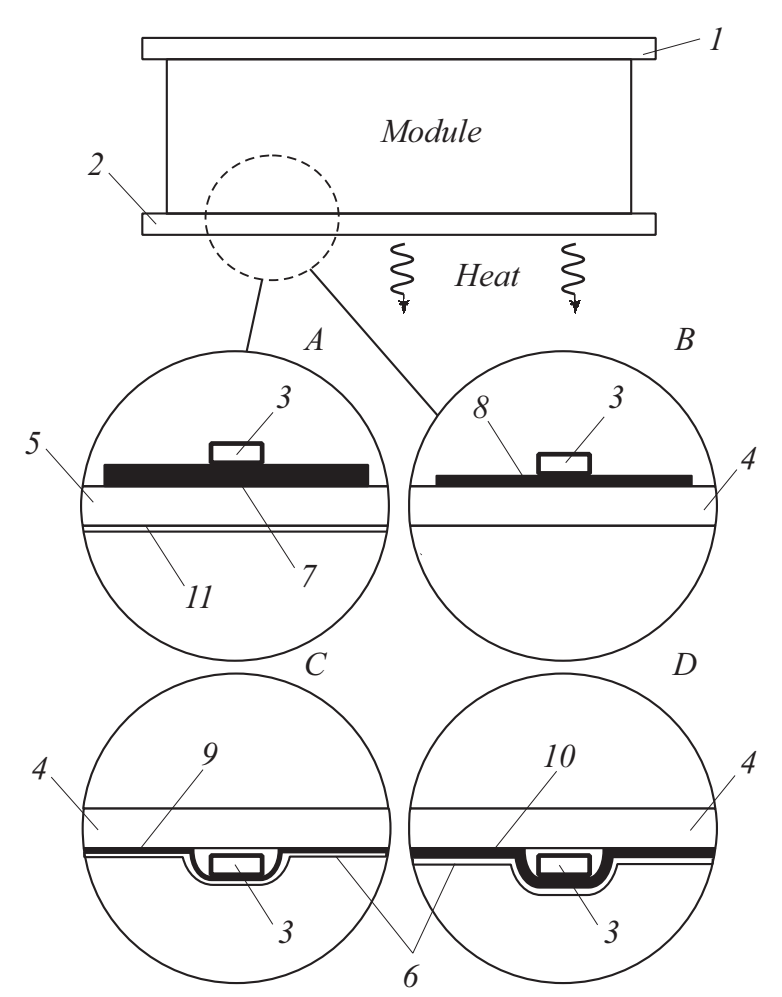

Рис. 3. Детали систем рассеивания тепла в фотоэлектрических концентраторных модулях типов $A, B, C$ и $D .1-$ линзовая панель, 2 - фотоприемная панель, 3 - фотопреобразователь, 4 - стеклянное основание, 5 - алюминиевая плита, 6 - ламинирующая пленка, 7 - алюмооксидная печатная плата, 8 - медь толщиной $1 \mathrm{~mm}, 9-$ медь толщиной $0.5 \mathrm{~mm}$, 10 - сталь толщиной $1 \mathrm{~mm}, 11$ - краска.

очередь, были закреплены на алюминиевой опорной плите толщиной $2 \mathrm{~mm}$ с покрашенной задней стороной для усиления процесса излучения тепла.
Остальные типы модулей имели приемные панели из стекла толщиной $4 \mathrm{~mm}$ в соответствии с концепциями „цельностеклянного“ и SMALFOC модуля $[1,4,8]$. Модули типа $B, C$ и $D$ предназначались для работы с линзами Френеля размерами $60 \times 60 \mathrm{~mm}$ с фокусным расстоянием $105 \mathrm{~mm}$. Фотоэлементы были припаяны к медным или стальным теплоотводам разной толщины (рис. 3), которые, в свою очередь, были закреплены на стеклянных опорных пластинах.

Линзовая панель модуля типа $B$ содержала шесть линз Френеля размерами $60 \times 60 \mathrm{~mm}$, фотоэлементы размерами $2.7 \times 2.7 \mathrm{~mm}$ были напаяны на медные теплоотводы размерами $40 \times 40 \mathrm{~mm}$ и толщиной $1 \mathrm{~mm}$, которые были размещены на внутренней стороне стеклянной пластины. Все фотоэлементы на приемных панелях в модулях типа $A$ и $B$ были электрически соединены последовательно.

Модули типа $C$ и $D$ содержали линзовые панели, состоящие из восьми линз Френеля размерами $60 \times 60 \mathrm{~mm}$. Фотоэлементы размерами $3 \times 3.5 \mathrm{~mm}$ были напаяны на единые теплоотводы, представляющие собой металлические профилированные пластины с углублениями под элементы, и электрически соединялись параллельно. Теплоотводы закреплялись на задней стороне стеклянной опорной пластины с помощью герметизирующей ламинирующей пленки [3]. Разница в конструкции модулей заключалась в материале и толщине теплоотводов: в модулях типа $C$ была использована медь толщиной $0.5 \mathrm{~mm}$; в модулях типа $D-$ сталь толщиной $1 \mathrm{~mm}$.

Конструктивно модули типа $C$ и $D$ являются единичными субмодулями исследованных полноразмерных фотоэлектрических концентраторных модулей. Полноразмерные модули состояли из линзовой панели с линзами Френеля размером $60 \times 60 \mathrm{~mm}$, общим количеством 128 штук (рис. 4, $a$ ) и приемной панели с соответствующим количеством солнечных элементов,

$a$
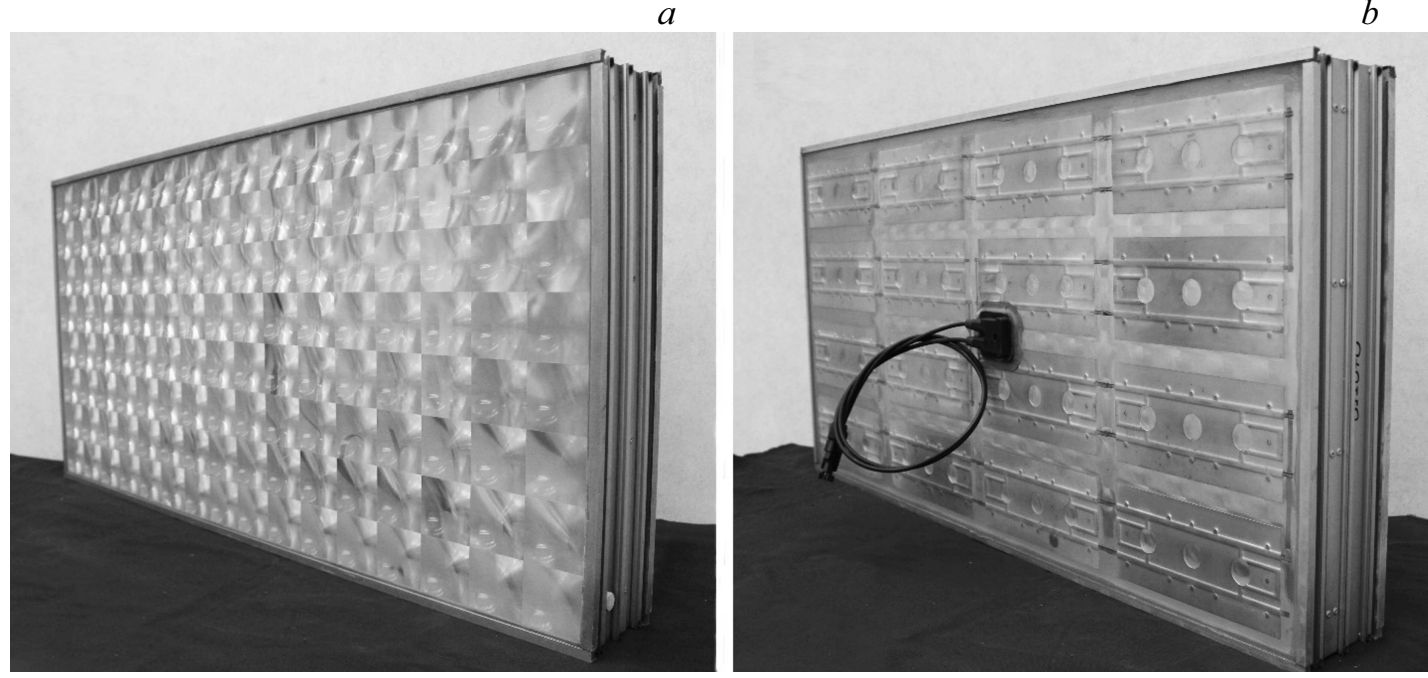

Рис. 4. Полноразмерный фотоэлектрический концентраторный модуль: $a-$ вид со стороны линзовой панели, $b-$ вид со стороны приемной панели. 
Таблица 1. Температура перегрева $\Delta T_{p-n}$ и эффективное тепловое сопротивление $r_{t h}$ для модуля типа $A$

\begin{tabular}{c|c|c|c|c|c|c|c}
\hline \multirow{2}{*}{ Плотность тока, $\mathrm{A} / \mathrm{cm}^{2}$} & \multirow{2}{*}{$\beta_{\text {cell }},-\mathrm{mV} /{ }^{\circ} \mathrm{C}$} & \multicolumn{3}{|c|}{ Температура перегрева, ${ }^{\circ} \mathrm{C}$} & \multicolumn{3}{|c}{ Тепловое сопротивление, ${ }^{\circ} \mathrm{C} \cdot \mathrm{m}^{2} / \mathrm{W}$} \\
\cline { 3 - 8 } & & $3.76 \mathrm{~W}$ & $7.52 \mathrm{~W}$ & $11.99 \mathrm{~W}$ & $3.76 \mathrm{~W}$ & $7.52 \mathrm{~W}$ & $11.99 \mathrm{~W}$ \\
\hline 0.5 & 5.27 & 9.5 & 18.4 & 28.0 & 0.049 & 0.047 & 0.045 \\
2 & 5.04 & 9.4 & 18.5 & 28.0 & 0.048 & 0.047 & 0.045 \\
3 & 4.78 & & 18.4 & 28.1 & & 0.047 & 0.045 \\
& 4.64 & & & 27.9 & & & 0.045
\end{tabular}

Таблица 2. Температура перегрева $\Delta T_{p-n}$ и эффективное тепловое сопротивление $r_{t h}$ для модуля типа $B$

\begin{tabular}{c|c|c|c|c|c}
\hline \multirow{2}{*}{ Плотность тока, $\mathrm{A} / \mathrm{cm}^{2}$} & \multirow{2}{*}{$\beta_{\text {cell }},-\mathrm{mV} /{ }^{\circ} \mathrm{C}$} & \multicolumn{2}{|c|}{ Температура перегрева, ${ }^{\circ} \mathrm{C}$} & \multicolumn{2}{|c}{ Тепловое сопротивление, ${ }^{\circ} \mathrm{C} \cdot \mathrm{m}^{2} / \mathrm{W}$} \\
\cline { 3 - 6 } & & $3.74 \mathrm{~W}$ & $14.92 \mathrm{~W}$ & $3.74 \mathrm{~W}$ & $14.92 \mathrm{~W}$ \\
\hline 0.5 & 5.40 & 13.2 & 44.3 & 0.075 & 0.064 \\
1 & 5.01 & 13.2 & 45.4 & 0.075 & 0.066 \\
2 & 4.76 & 12.6 & 45.7 & 0.073 & 0.066 \\
3 & 4.62 & & 45.9 & & 0.066 \\
6 & 4.38 & & 46.5 & & 0.067
\end{tabular}

объединенными в группы $16 \times 8$ штук. Каждая группа состояла из 8 элементов, напаянных на общем металлическом теплоотводе, электрически соединенных параллельно. 16 теплоотводов с установленными на них элементами были соединены последовательно. Теплоотводы представляют собой металлические профилированные пластины, изготовленные из листовой меди толщиной $0.5 \mathrm{~mm}$, либо стали толщиной $1 \mathrm{~mm}$. Теплоотводы с установленными на них элементами и соединяющими их электрическими шинами закреплены на наружной стороне задней стеклянной панели и загерметизированы ламинирующей пленкой. Задняя сторона приемной панели с закрепленными теплоотводами показана на рис. 4, $b$.

\section{3. Результаты и обсуждения}

Для каждого типа исследуемых модулей на этапе 1 в лабораторном термостате были определены температурные коэффициенты напряжения модулей $\beta_{\text {mod }}$ при различных плотностях тока элементов в диапазоне температур $T=20-80^{\circ} \mathrm{C}$. На этапе 2 модули размещались в открытом пространстве лаборатории и производился разогрев элементов за счет пропускания постоянного электрического тока в прямом направлении. Для каждого значения подводимой электрической мощности $P_{E l}$ на клеммах модуля были измерены значения напряжения $V_{a m b}$ и $V_{\text {hot }}$ до начала разогрева и после достижения теплового баланса с окружающей средой. Используя формулы (2) и (3), был произведен расчет температуры перегрева $p-n$-переходов элементов $\Delta T_{p-n}$ и эффективного теплового сопротивления $r_{t h}$.

В табл. 1-4 обобщены экспериментальные результаты по определению $r_{t h}$ для исследуемых модулей типа $A, B, C$ и $D$. В первых двух столбцах таблиц приведены значения плотности тока через элементы и соответствующие значения $\beta_{\text {cell }}$ элементов. Значения $\beta_{\text {cell }}$ рассчитывается путем деления $\beta_{\text {mod }}$ на количество элементов или групп элементов в каждом модуле при последовательном соединении элементов или групп.

Хотелось бы обратить внимание на хорошую повторяемость значений параметров $\Delta T_{p-n}$ и $r_{t h}$, даже если плотность тока при быстрых измерениях ВАХ изменяется на один порядок величины. Также следует отметить некоторое уменьшение параметра $r_{t h}$ при увеличении подводимой электрической мощности $P_{E l}$. Это можно объяснить тем, что повышение температуры во всех частях испытываемого модуля сопровождается повышением эффективности радиационного излучения и интенсификацией процесса рассеяния тепла за счет воздушной конвекции. Особенно наглядно эти эффекты проявляются в модулях типа $A$ и $B$ с последовательным соединением элементов. В модулях такой конструкции это может служить дополнительным подтверждением отсутствия неконтролируемых тепловых эффектов в используемом подходе при оценке температур в лабораторных условиях. В модулях типа $C$ и $D$ с параллельным соединением элементов при увеличении тока его распределение по элементам за счет некоторых различий в характеристиках элементов и подводящих шин становится неравномерным (в табл. 3 и 4 приведены усредненные значения плотности тока через элементы). Это приводит к неравномерному нагреву элементов, изменению условий отвода тепла и соответственно значений параметра $r_{t h}$.

В модуле типа $A$ эффективное тепловое сопротивления наиболее низкое, что объясняется изготовлением в этом модуле теплоотводящих деталей из алюминия, обладающего высокой теплопроводностью, и использо- 
Таблица 3. Температура перегрева $\Delta T_{p-n}$ и эффективное тепловое сопротивление $r_{t h}$ для модуля типа $C$ с медными теплоотводами

\begin{tabular}{|c|c|c|c|c|c|c|c|}
\hline \multirow{2}{*}{ Плотность тока, A/cm² } & \multirow{2}{*}{$\beta_{\text {cell }},-\mathrm{mV} /{ }^{\circ} \mathrm{C}$} & \multicolumn{3}{|c|}{ Температура перегрева, ${ }^{\circ} \mathrm{C}$} & \multicolumn{3}{|c|}{ Тепловое сопротивление, ${ }^{\circ} \mathrm{C} \cdot \mathrm{m}^{2} / \mathrm{W}$} \\
\hline & & $3.75 \mathrm{~W}$ & $7.59 \mathrm{~W}$ & $15.25 \mathrm{~W}$ & $3.75 \mathrm{~W}$ & $7.59 \mathrm{~W}$ & $15.25 \mathrm{~W}$ \\
\hline 0.60 & 5.22 & 9.1 & 19.6 & 37.1 & 0.070 & 0.074 & 0.070 \\
\hline 0.95 & 5.04 & 9.2 & 20.1 & 37.7 & 0.070 & 0.076 & 0.071 \\
\hline 1.19 & 4.93 & 8.5 & 20.1 & 37.8 & 0.065 & 0.076 & 0.071 \\
\hline 1.49 & 4.82 & 9.7 & 22.2 & 40.1 & 0.074 & 0.084 & 0.076 \\
\hline 1.79 & 4.74 & & 22.4 & 40.7 & & 0.085 & 0.077 \\
\hline 2.08 & 4.66 & & 22.4 & 41.0 & & 0.085 & 0.077 \\
\hline 2.38 & 4.58 & & 21.5 & 41.0 & & 0.082 & 0.078 \\
\hline 2.98 & 4.46 & & 20.7 & 42.4 & & 0.078 & 0.080 \\
\hline 3.57 & 4.34 & & & 42.1 & & & 0.080 \\
\hline 4.17 & 4.24 & & & 43.5 & & & 0.082 \\
\hline 4.76 & 4.15 & & & 42.8 & & & 0.081 \\
\hline
\end{tabular}

Таблица 4. Температура перегрева $\Delta T_{p-n}$ и эффективное тепловое сопротивление $r_{t h}$ для модуля типа $D$ со стальными теплоотводами

\begin{tabular}{|c|c|c|c|c|c|c|c|}
\hline \multirow{2}{*}{ Плотность тока, A/ $\mathrm{cm}^{2}$} & \multirow{2}{*}{$\beta_{\text {cell }},-\mathrm{mV} /{ }^{\circ} \mathrm{C}$} & \multicolumn{3}{|c|}{ Температура перегрева, ${ }^{\circ} \mathrm{C}$} & \multicolumn{3}{|c|}{ Тепловое сопротивление, ${ }^{\circ} \mathrm{C} \cdot \mathrm{m}^{2} / \mathrm{W}$} \\
\hline & & $3.79 \mathrm{~W}$ & $7.53 \mathrm{~W}$ & $14.95 \mathrm{~W}$ & $3.79 \mathrm{~W}$ & $7.53 \mathrm{~W}$ & $14.95 \mathrm{~W}$ \\
\hline 0.60 & 4.99 & 9.5 & 20.1 & 42.8 & 0.073 & 0.077 & 0.082 \\
\hline 1.01 & 4.82 & 9.4 & 20.0 & 42.4 & 0.072 & 0.077 & 0.082 \\
\hline 1.61 & 4.69 & 10.0 & 20.7 & 42.8 & 0.076 & 0.079 & 0.082 \\
\hline 2.02 & 4.65 & 10.1 & 21.0 & 42.9 & 0.077 & 0.080 & 0.083 \\
\hline 2.52 & 4.59 & 10.2 & 21.2 & 43.1 & 0.078 & 0.081 & 0.083 \\
\hline 3.02 & 4.52 & & 21.5 & 43.4 & & 0.082 & 0.084 \\
\hline 3.53 & 4.50 & & 21.7 & 43.6 & & 0.083 & 0.084 \\
\hline 4.03 & 4.46 & & 21.7 & 43.6 & & 0.083 & 0.084 \\
\hline 5.04 & 4.41 & & & 44.2 & & & 0.085 \\
\hline 6.05 & 4.34 & & & 44.5 & & & 0.086 \\
\hline 7.06 & 4.28 & & & 44.6 & & & 0.086 \\
\hline 8.06 & 4.99 & & & 44.5 & & & 0.086 \\
\hline
\end{tabular}

ванием краски для увеличения рассеяния тепла. В модуле типа $B$ стеклянная платина приемной панели делает процесс рассеяния тепла более трудным, несмотря на использование медных теплоотводов толщиной $1 \mathrm{~mm}$. В модулях типов $C$ и $D$ стеклянные основания играют небольшую роль, потому что металлические теплоотводы с фотоэлементами помещены на наружной стороне стеклянных платин. Тепловое сопротивление в модуле типа $C$, несмотря на использование медных теплоотводов большей площади, немного выше по сравнению с типом $B$ за счет низкой теплопроводности пленки ламинирующего покрытия. Определенное увеличение теплового сопротивления в случае модуля типа $D$ связано с использованием стали вместо меди для теплоотводящих пластин.

Тем не менее положительные особенности конструкции последнего модуля могут иметь важное значение, поскольку основные конструкционные материалы весьма дешевы, а также сталь и стекло как конструкционные материалы более совместимы по коэффициентам теплового расширения [3].

Полноразмерные фотоэлектрические концентраторные модули с медными или стальными теплоотводами подвергались такой же процедуре при определении $r_{t h}$, как было описано выше для малых модулей. Отличие состояло в том, что при определении $\beta_{\text {mod }}$ была использована термостатная камера больших размеров. Входная электрическая мощность для нагрева модулей на втором этапе измерений выбиралась минимально возможной для предотвращения неравномерности распределения прямого тока по элементам. Были выбраны два значения $P_{E l}$, удовлетворяющих этим требованиям, 20 и $30 \mathrm{~W}$ (при этом плотность мощности была ниже самой низкой, используемой при получении результатов, приведенных в табл. 1-4). При проведении исследований зависимости значений $r_{t h}$ от пространственного расположения модулей были выбраны три наиболее характерных рабочих положения модулей при преоб- 
Таблица 5. Значения эффективных тепловых сопротивлений $r_{t h}$ полноразмерного фотоэлектрического концентраторного модуля с медными теплоотводами, толщиной $0.5 \mathrm{~mm}$

\begin{tabular}{|c|c|c|c|c|c|c|c|}
\hline \multirow{4}{*}{ Плотность тока, A/cm ${ }^{2}$} & \multirow{4}{*}{$\beta_{\text {cell }},-\mathrm{mV} /{ }^{\circ} \mathrm{C}$} & \multicolumn{6}{|c|}{ Тепловое сопротивление, ${ }^{\circ} \mathrm{C} \cdot \mathrm{m}^{2} / \mathrm{W}$} \\
\hline & & \multicolumn{6}{|c|}{ Положение модуля } \\
\hline & & \multicolumn{2}{|c|}{ Вертикальное } & \multicolumn{2}{|c|}{ Наклон $45^{\circ}$} & \multicolumn{2}{|c|}{ Горизонтальное } \\
\hline & & $20 \mathrm{~W}$ & $30 \mathrm{~W}$ & $20 \mathrm{~W}$ & $30 \mathrm{~W}$ & $20 \mathrm{~W}$ & $30 \mathrm{~W}$ \\
\hline 0.14 & 5.96 & 0.082 & 0.080 & 0.080 & 0.081 & 0.082 & 0.087 \\
\hline 0.20 & 5.74 & 0.081 & 0.081 & 0.083 & 0.084 & 0.084 & 0.088 \\
\hline 0.27 & 5.59 & 0.080 & 0.083 & 0.082 & 0.085 & 0.082 & 0.090 \\
\hline 0.55 & 5.31 & 0.082 & 0.082 & 0.082 & 0.084 & 0.084 & 0.089 \\
\hline 0.82 & 5.11 & & 0.082 & & 0.085 & & 0.086 \\
\hline
\end{tabular}

Таблица 6. Значения эффективных тепловых сопротивлений $r_{t h}$ полноразмерного фотоэлектрического концентраторного модуля со стальными теплоотводами, толщиной $1 \mathrm{~mm}$

\begin{tabular}{|c|c|c|c|c|c|c|c|}
\hline \multirow{4}{*}{ Плотность тока, $\mathrm{A} / \mathrm{cm}^{2}$} & \multirow{4}{*}{$\beta_{\text {cell }},-\mathrm{mV} /{ }^{\circ} \mathrm{C}$} & \multicolumn{6}{|c|}{ Тепловое сопротивление, ${ }^{\circ} \mathrm{C} \cdot \mathrm{m}^{2} / \mathrm{W}$} \\
\hline & & \multicolumn{6}{|c|}{ Положение модуля } \\
\hline & & \multicolumn{2}{|c|}{ Вертикальное } & \multicolumn{2}{|c|}{ Наклон $45^{\circ}$} & \multicolumn{2}{|c|}{ Горизонтальное } \\
\hline & & $20 \mathrm{~W}$ & $30 \mathrm{~W}$ & $20 \mathrm{~W}$ & $30 \mathrm{~W}$ & $20 \mathrm{~W}$ & $30 \mathrm{~W}$ \\
\hline 0.14 & 5.43 & 0.086 & 0.091 & 0.097 & 0.096 & 0.096 & 0.101 \\
\hline 0.20 & 5.33 & 0.081 & 0.089 & 0.097 & 0.097 & 0.092 & 0.100 \\
\hline 0.27 & 5.15 & 0.085 & 0.092 & 0.099 & 0.096 & 0.092 & 0.101 \\
\hline 0.55 & 4.95 & 0.088 & 0.093 & 0.100 & 0.098 & 0.093 & 0.102 \\
\hline 0.82 & 4.88 & & 0.092 & & 0.098 & & 0.101 \\
\hline
\end{tabular}

разовании солнечного излучения в натурных условиях. Полноразмерные фотоэлектрические концентраторные модули последовательно устанавливались в вертикальное положение, под углом $45^{\circ}$ к горизонтальной плоскости теплоотводами вниз и в горизонтальное положение теплоотводами вниз. Соответствующие результаты приведены в табл. 5 и 6.

Как видно из приведенных результатов, существует заметная разница в значениях $r_{t h}$ для модулей, в которых теплоотводы изготавливаются из меди и стали. Так же можно отметить, что процесс рассеивания тепла меняется, когда модули вращаются от вертикального положения к горизонтальному. В горизонтальном положении теплоотводами вниз конвекция воздуха вдоль задней стороны корпуса модуля наиболее затруднена.

Результаты, приведенные в табл. 5 и 6, находятся в хорошем согласии с результатами для модулей малых размеров аналогичной конструкции. Следует учитывать, что в полноразмерных модулях получены несколько более высокие значения $r_{t h}$, определенные при малой входной электрической мощности. При низком перегреве корпусов модулей процессы конвекции воздуха действительно значительно слабее. Более того, в меньших по размеру модулях отношение площади боковых стенок модуля к площади активной области тепловыделения было заметно больше по сравнению с полноразмерны- ми модулями, что способствовало более интенсивному переносу тепла внутри модулей от фотоэлементов к боковым стенкам. В результате тепловое сопротивление фотоэлемент-окружающая среда в случае больших модулей оказалось несколько выше.

\section{Заключение}

Эффективное тепловое сопротивление $r_{t h}$ фотоэлемент-окружающая среда фотоэлектрического концентраторного модуля является общей тепловой характеристикой, которая позволяет сравнивать модули разных конструкций по эффективности рассеивания тепла в различных условиях окружающей среды. В работе использована методика для исследования тепловых свойств модулей в лабораторных условиях при разогреве элементов за счет пропускания постоянного тока. Лабораторные условия характеризуются стабильностью в организации измерений, так что при относительно простой процедуре оценки $r_{t h}$ может быть выявлено влияние некоторых структурных деталей конструкции и пространственного положения модуля на эффективность теплосброса. В работе данная методика применена для исследования тепловых свойств модулей, различающихся по размерам и материалам теплоотводов. Также было 
исследовано влияние пространственного положения модуля на условия отвода тепла от фотоэлементов. В разработанной методике исключены неопределенности, связанные с расположением датчиков температуры и нестабильностью метеорологических условий при проведении аналогичных исследований в натурных условиях.

\section{Конфликт интересов}

Авторы заявляют, что у них нет конфликта интересов.

\section{Список литературы}

[1] M. Muller, C. Deline, B. Marion, S. Kurtz, N. Bosco. AIP Conf. Proc., 1407, 331 (2011). DOI: 10.1063/1.3658355

[2] V.D. Rumyantsev, A.V. Chekalin, N.Yu. Davidyuk, D.A. Malevskiy, P.V. Pokrovskiy, N.A. Sadchikov, A.N. Panchak. AIP Conf. Proc., 1556, 138 (2013). DOI: $10.1063 / 1.4822217$

[3] V.D. Rumyantsev, V.M. Andreev, A.V. Chekalin, N.Yu. Davidyuk, O.A. Im, E.V. Khazova, N.A. Sadchikov. AIP Conf. Proc., 1556, 185 (2013). DOI: 10.1063/1.4822227

[4] V.D. Rumyantsev, A.V. Chekalin, N.Yu. Davidyuk, D.A. Malevskiy, M.Z. Shvarts, A. Luque, V.M. Andreev. AIP Conf. Proc., 1616, 154 (2014). DOI: 10.1063/1.4897050

[5] V.D. Rumyantsev, A.V. Chekalin, N.Yu. Davidyuk, N.A. Sadchikov, A. Luque. Prog. Photovolt: Res. Appl., 24 (2), 211 (2015). DOI: 10.1002/pip.2662

[6] R. Núñez, I. Antón, S. Askins, G. Sala, K. Araki. AIP Conf. Proc., 1616, 144 (2014). DOI: 10.1063/1.4897048

[7] V.D. Rumyantsev, N.Yu. Davidyuk, E.A. Ionova, P.V. Pokrovskiy, N.A. Sadchikov, V.M. Andreev. AIP Conf. Proc., 1277, 89 (2010). DOI: 10.1063/1.3509240

[8] G. Timo, A. Minuto, P. Groppelli, E. Malvisi, G. Smekens, M. Noack, M. Sturm, K. Khalaidovski. Proc. of the 24th EU PVSEC, 21 (2009).

[9] V.D. Rumyantsev, V.M. Andreev, A.V. Chekalin, N.Yu. Davidyuk, N.A. Sadchikov. 2014 IEEE 40th Photovolt. Specialist Conf. (PVSC), 2720 (2014). DOI: 10.1109/PVSC.2014.6925491

[10] Y. Xi, E.F. Schubert. Appl. Phys. Lett., 85, 2163 (2004). DOI: $10.1063 / 1.1795351$ 